\title{
Влияние деформации сжатия и растяжения на спектр дислокационной люминесценции в кремнии
}

\author{
(C) Н.А. Соболев ${ }^{1}$, А.Е. Калядин ${ }^{1}$, О.В. Феклисова ${ }^{2}$, Е.Б. Якимов ${ }^{2}$ \\ ${ }^{1}$ Физико-технический институт им. А.Ф. Иоффее Российской академии наук, \\ 194021 Санкт-Петербург, Россия \\ ${ }^{2}$ Институт проблем технологии микроэлектроники и особочистых материалов Российской академии наук, \\ 142432 Черноголовка, Россия \\ E-mail: nick@sobolev.ioffe.rssi.ru
}

Поступила в Редакцию 17 марта 2021 г.

В окончательной редакции 25 марта 2021 г.

Принята к публикации 25 марта 2021 г.

Исследована фотолюминесценция в кремнии, деформированном методом четырехточечного изгиба при температуре $600^{\circ} \mathrm{C}$. С обеих сторон деформированных образцов наблюдаются так называемые линии дислокационной люминесценции D1, D2, D3 и D4. Обнаружено, что для образцов с плотностью введенных дислокаций $\sim 10^{7} \mathrm{~cm}^{-2}$ интенсивность люминесценции линий D3 и D4 одинаковая с обеих сторон образцов, а интенсивности линий D1 и D2 со стороны растяжения выше, чем со стороны сжатия. Поведение интенсивности линий D1 и D2 хорошо коррелирует с количеством следов за дислокациями. Обсуждаются возможные причины наблюдавшегося эффекта.

Ключевые слова: дислокационная люминесценция, кремний, метод четырехточечного изгиба.

DOI: 10.21883/FTP.2021.07.51014.9651

Дислокационная фотолюминесценция (ФЛ) впервые наблюдалась в [1] в пластинах кремния, деформированных при $850^{\circ} \mathrm{C}$ методом четырехточечного изгиба. Были обнаружены 4 линии люминесценции: D1 (0.812 эВ), D2 (0.875 эВ), D3 (0.934 эВ) и D4 (1.000 эВ). Интенсивность этих линий оказалась пропорциональной плотности дислокаций, что позволило авторам связать их образование с дислокациями. В следующей работе этих же авторов было установлено, что природа двух пар линий D1/D2 и D3/D4 различная, поскольку под влиянием одноосного сжатия сдвиги этих линий в каждой паре аналогичны, но различаются для обеих пар [2]. В работе [3] было исследовано влияние термообработки и одноосного сжатия на спектры ФЛ деформированных образцов. При последующей деформации при низкой температуре и больших напряжениях линии D1-D4 исчезали и образовывались новые линии D5 и D6. При последующем отжиге без приложения напряжения линии D5 и D6 отжигались и восстанавливались линии D1-D4, но изменялось соотношение их интенсивностей. При изучении влияния одноосного сжатия для различных кристаллографических направлений на энергетическое положение линий D1/D2 и D3/D4 в [3] было обнаружено их расщепление, а не только сдвиг, как это наблюдалось в [2]. Люминесцентные и структурные (использован метод просвечивающей электронной микроскопии) свойства исследовались параллельно, что дало возможность изучать не только трансформацию структурных дефектов, но и их роль в образовании люминесцентных центров. Полученные результаты позволили авторам [3] предположить, что образование центров D3/D4 обусловлено свойствами самих дислокаций, а центров D1/D2 - точечными дефектами, образующи- мися в полях напряжений дислокаций. В работах $[4,5]$ изучалась дислокационная электролюминесценция (ЭЛ) в образцах с $p-n$-переходами, подвергнутых четырехточечному изгибу. При такой деформации на одной широкой плоскости образца действует напряжение сжатия, а на противоположной - напряжение растяжения. В [4,5] $p$-n-переходы располагались со стороны растяжения, с которой измерялась люминесценция. Структуры с $p^{+}-n-$ и $n^{+}-p$-переходами формировались на кремнии, выращенном методами бестигельной зонной плавки $(F Z-\mathrm{Si})$ и Чохральского $(C z-\mathrm{Si})$, с помощью имплантации ионов В и $\mathrm{P}$ соответственно и последующих отжигов. После изгиба в спектрах люминесценции доминировали линии D1-D4. Максимальная интенсивность линий наблюдалась в образцах, выращенных методом FZ-Si и прошедших постимплантационный отжиг при наименьшей температуре. В этих образцах интенсивности линий снижались в последовательности D4, D3, D2 и D1. Таким образом, в образцах, деформированных методом изгиба, во всех опубликованных работах свойства линий дислокационной люминесценции в зависимости от технологических условий (тип кремния, ориентация изгибаемых пластин, температура изгиба, условия изготовления $p-n$-переходов и т.д.) и условий измерения (температура образца, длина волны и мощность лазера, прикладываемое одноосное давление, ток через $p-n$ переход и т.д.) либо исследовались только со стороны растяжения [4,5], либо знак действовавших напряжений не указывался [1-3]. Таким образом, исследования влияния знака деформации на спектр ФЛ ранее не проводились. В то же время в проведенных исследованиях электрически активных центров с помощью методов EBIC и LBIC в деформированных таким же способом 
образцах было установлено, что рекомбинационная активность точечных дефектов, остающихся в плоскости скольжения дислокаций (dislocation trails), различается со стороны сжатия и растяжения [6-9]. Спектры DLTS, измеренные на сторонах сжатия и растяжения, также различались [10]. Поэтому цель данной работы заключалась в сравнительных исследованиях спектров ФЛ, измеренных на сжатой и растянутой сторонах образца, деформированного четырехточечным изгибом.

Образцы, вырезанные из кремния $n$-типа проводимости с удельным сопротивлением 40 Ом · см, выращенного методом бестигельной зонной плавки, деформировали методом четырехточечного изгиба вокруг оси $\langle 112\rangle$ (плоскость поверхности была $\{111\}$, а боковые грани $\{112\}$ и $\{110\})$. Деформация проводилась при $600^{\circ} \mathrm{C}$ в течение 4 ч и нагрузке 2.5 кг/мм ${ }^{2}$. Источником дислокаций служила царапина, нанесенная алмазной иглой. Плотность дислокаций, измеренная с обеих сторон после селективного химического травления, различалась незначительно и составляла $\sim 10^{7} \mathrm{~cm}^{-2}$. После деформации в образцах с указанной выше ориентацией введенные дислокационные полупетли содержат два $60^{\circ}$-х и один винтовой сегменты. При этом один из сегментов, пересекающих поверхность, перпендикулярен действующей силе и заведомо является $60^{\circ}$-м, а второй пересекающий поверхность сегмент может быть как винтовым, так и $60^{\circ}$-м. ФЛ возбуждалась излучением твердотельного лазера с длиной волны 532 нм, мощностью $0.9-36$ мВт и измерялась при $5 \mathrm{~K}$.

Спектры ФЛ со стороны растяжения при разной мощности накачки приведены на рис. 1. С ростом мощности накачки интенсивность линий люминесценции нарастает. Сравнение нормированных спектров показало, что они практически совпадают, т.е. форма спектров не изменяется при увеличении мощности накачки. В спектрах

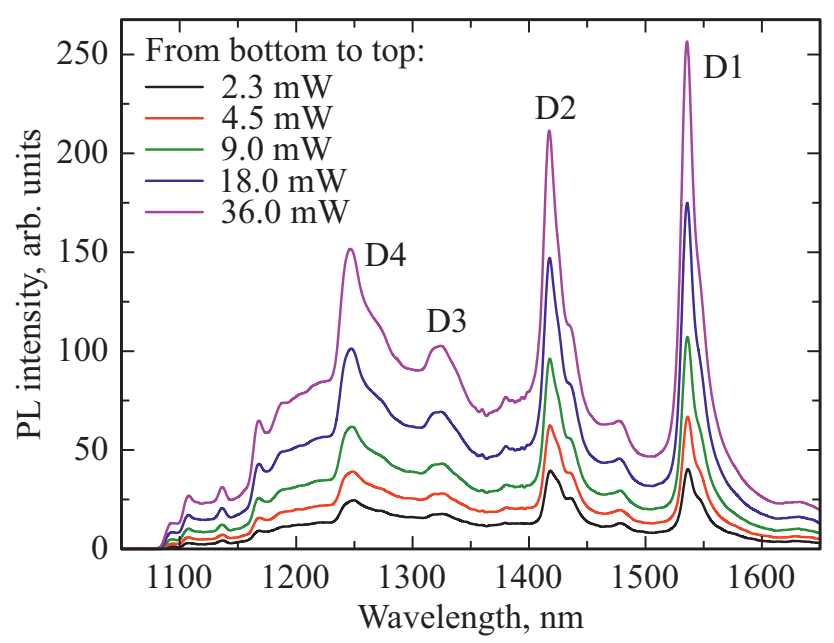

Рис. 1. Влияние мощности накачки на спектры фотолюминесценции (PL) со стороны растяжения при $5 \mathrm{~K}$. Диаметр луча лазера 2 мм, разрешение установки 5.4 нм. (Цветной вариант рисунка представлен в электронной версии статьи).

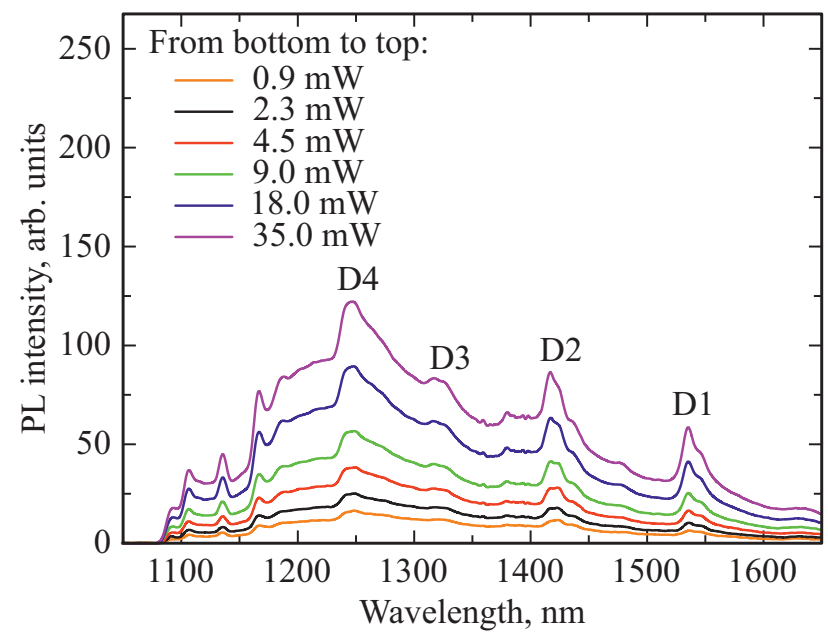

Рис. 2. Влияние мощности накачки на спектры фотолюминесценции (PL) со стороны сжатия при $5 \mathrm{~K}$. Диаметр луча лазера 2 мм, разрешение установки 5.4 нм.

доминируют четыре линии дислокационной люминесценции: D1 (1536 нм), D2 (1418 нм), D3 (1317 нм) и D4 (1241 нм). Важно отметить, что интенсивности линий D1 и D2 превышают интенсивности линий D3 и D4. Положение линий в точности совпадает с положением линий, наблюдавшихся в деформированных одноосным сжатием образцах в работе [3], в которой использовались другие марки кремния и другие условия деформации.

Спектры ФЛ со стороны сжатия при разной мощности накачки приведены на рис. 2. Положение линий D1-D4 не изменяется по сравнению с их положением при измерении со стороны растяжения. Как и в предыдущем случае, при увеличении мощности накачки форма линий ФЛ не изменяется. При одинаковой мощности накачки интенсивности линий D3 и D4 с обеих сторон пластины практически совпадают. Существенное различие заключается в том, что для напряжений сжатия интенсивности линий D1 и D2 меньше, чем интенсивности линий D3 и D4. Ранее такое соотношение наблюдалось при низкой плотности дислокаций, а при высокой плотности интенсивность линий D1 и D2 превышала интенсивность линий D3 и D4 [3,11].

Обычно считают, что линии D3 и D4 обусловлены расщепленными $60^{\circ}$-ми дислокациями $[3,11]$. В исследованных образцах плотность дислокаций и количество $60^{\circ}$-х сегментов вблизи поверхности примерно равны с обеих сторон. Поэтому равенство интенсивностей линий D3 и D4 на сторонах сжатия и растяжения легко объяснимо.

С объяснением различия интенсивностей линий D1 и D2 ситуация более сложная. Их природа окончательно не установлена до сих пор, а механизм образования центров D1 и D2 в случае деформации образцов методом четырехточечного изгиба, т.е. при достаточно низкой плотности дислокаций и достаточно низких температурах, может отличаться от механизмов, реализуемых 
при других методах введения дислокаций. Как показано в [12], в случаях термического отжига имплантированного кремния при высокой температуре в условиях пересыщения кремния собственными межузельными атомами [12] или эпитаксиального роста слоев $\mathrm{SiGe}$ на пластине $\mathrm{Si}$ [13] сначала образуются петли Франка, затем часть этих петель преобразуется в совершенные призматические дислокационные петли, а многократные пересечения совершенных петель приводят к образованию трехмерной цепочки с высокой плотностью чисто краевых дислокаций (pure edge dislocations), которые и связаны с линией D1. Недавно были получены экспериментальные данные, уточняющие связь линий D1 и D2 с конкретными структурными дефектами при отжиге имплантированных образцов [14]. Авторы утверждали, что линия D1 непосредственно связана с ядром дислокации Ломера. Представления о природе центра D2 еще менее определенны по сравнению с центром D1. Так, в уже упомянутой работе [14] авторы считают, что линия D2 связана с метастабильными межузельными микродефектами $\{111\},\{113\}$ и $\{001\}$, образующимися вблизи ядра дислокации.

При низкой плотности дислокаций образование большого количества дислокаций Ломера представляется маловероятным. Приложенные напряжения также представляются слишком низкими для существенного влияния на процессы перестройки дефектов. Поэтому причину различия спектров, по-видимому, следует искать исходя из результатов исследования электрически активных следов, остающихся в результате скольжения $60^{\circ}$-х дислокаций (dislocation trails) [6-10,15,16]. В этих работах методами EBIC и LBIC было показано, что в использованной в настоящей работе ориентации образцов на стороне растяжения электрически активные следы образуются за заведомо $60^{\circ}$-м сегментом дислокационной полупетли, пересекающим поверхность [7-9]. На стороне сжатия следы формируются за другим пересекающим поверхность концом полупетли или за ее дном. Таким образом, в этих работах также наблюдалось различие между сторонами сжатия и растяжения. Анализ, проведенный в [15], показал, что следы формируются только за $60^{\circ}$-ми дислокациями, у которых лидирующими являются $90^{\circ}$-е частичные дислокации. Таким образом, на стороне растяжения во всех полупетлях вблизи поверхности присутствует $60^{\circ}$-я дислокация с лидирующей $90^{\circ}$-й частичной дислокацией. На стороне сжатия такая дислокация равновероятно может либо выходить на поверхность, либо являться дном полупетли и располагаться на глубине, превышающей 100 мкм. Поскольку глубина проникновения света от лазера $\sim 1.5$ мкм, дно дислокационной полупетли не может вносить вклад в сигнал ФЛ. Из этого следует, что даже при одинаковой плотности дислокаций чисто статистически количество следов за дислокациями, а также количество $60^{\circ}$-х дислокаций с лидирующей $90^{\circ}$-й частичной дислокацией в области возбуждения лазера на стороне растяжения должно быть в 2 раза больше, чем на стороне сжатия. Таким образом, наблюдается явная корреляция между количеством следов за дислокациями и интенсивностью линий D1 и D2. Однако мало вероятно, что следы за дислокациями сами по себе связаны с ФЛ, поскольку они стабильны только до температур $\sim(800-850)^{\circ} \mathrm{C}[6,17]$, в то время как линии D1 и D2 наблюдаются и после отжига при значительно более высоких температурах [12].

Формирование следов за дислокациями не может происходить без участия собственных точечных дефектов, генерируемых движущимися дислокациями. Кроме того, как показано в [18], порядок следования частичных дислокаций может влиять на структуру их ядра. Об этом, по-видимому, свидетельствует и то, что заметные следы формируются только за расщепленной $60^{\circ}$-й дислокацией с лидирующей $90^{\circ}$-й дислокацией [15]. Исходя из этого можно предположить, что если при движении дислокаций генерируются междоузлия, то с их участием могут формироваться дефекты, аналогичные образующимся при отжиге имплантированных образцов. Формирование протяженных дефектов в плоскостях скольжения за движущимися дислокациями было выявлено в [19] избирательным химическим травлением. Второе возможное объяснение может быть связано с разной структурой ядра расщепленных $60^{\circ}$-х дислокаций с разным типом лидирующей частичной дислокации и влиянием этой структуры на интенсивность линий D1 и D2.

Таким образом, в работе проведено сравнение спектров дислокационной ФЛ на растянутой и сжатой поверхностях одной и той же пластины кремния, деформированной методом четырехточечного изгиба. Установлено, что интенсивности линий D3 и D4 практически одинаковы с обеих сторон образца при одинаковой мощности возбуждения ФЛ. Это обусловлено тем, что на разных сторонах пластины плотности расщепленных дислокаций, с которыми связывают центры D3 и D4, незначительно отличаются друг от друга. Интенсивности линий D1 и D2 со стороны растяжения выше, чем со стороны сжатия. Поведение интенсивности этих линий хорошо коррелирует с количеством следов, формирующихся за движущимися дислокациями. Это позволяет предположить, что на интенсивность линий D1 и D2 влияют структура ядра $60^{\circ}$-й дислокации либо кластеры собственных точечных дефектов, генерируемых дислокациями.

\section{Финансирование работы}

Работа О.В. Феклисовой и Е.Б. Якимова частично выполнена в рамках государственного задания 075-0035521-00.

\section{Конфликт интересов}

Авторы заявляют, что у них нет конфликта интересов. 


\section{Список литературы}

[1] Н.А. Дроздов, А.А. Патрин, В.Д. Ткачев. Письма ЖЭТФ, 23, 651 (1976).

[2] N.A. Drozdov, A.A. Patrin, V.D. Tkachev: Phys. Status Solidi B, 83, K137 (1977).

[3] R. Sauer, J. Weber, J. Stolz, E.R. Weber, K.H. Kurster, H. Alexander. Appl. Phys. A, 36, 1 (1985).

[4] N.A. Sobolev, A.M. Emel'yanov, E.I. Shek, O.V. Feklisova, E.B. Yakimov, T.V. Kotereva. Phys. Status Solidi C, 2, 1842 (2005).

[5] Н.А. Соболев, А.М. Емельянов, Е.И. Шек, О.В. Феклисова, Е.Б. Якимов. ФТП, 39 (10), 1271 (2005).

[6] I.E. Bondarenko, V.G. Eremenko, B.Ya. Farber, V.I. Nikitenko, E.B. Yakimov. Phys. Status Solidi A, 68, 53 (1981).

[7] I.E. Bondarenko, H. Blumtritt, J. Heydenreich, V.V. Kazmiruk, E.B. Yakimov. Phys. Status Solidi A, 95, 173 (1986).

[8] O.V. Feklisova, V.I. Orlov, E.B. Yakimov. Phys. Status Solidi C, 12 (8), 1081 (2015).

[9] V.I. Orlov, E.B. Yakimov, N. Yarykin. Sol. St. Phenomena, 242, 155 (2016).

[10] O.A. Soltanovich, V.I. Orlov, N. Yarykin, E.B. Yakimov. Phys. Status Solidi A, 216, 1900326 (2019).

[11] V. Kveder, M. Kittler. Mater. Sci. Forum, 590, 29 (2008).

[12] N.A. Sobolev, O.B. Gusev, E.I. Shek, V.I. Vdovin, T.G. Yugova, A.M. Emel'yanov. Appl. Phys. Lett., 72, 3326 (1998).

[13] S. Fukatsu, Y. Mera, M. Inoue, K. Maeda, H. Akiyama, H. Sakaki. Appl. Phys. Lett., 68, 1889 (1996).

[14] L.I. Fedina, A.K. Gutakovskii, T.S. Shamirzaev. J. Appl. Phys., 124, 053106 (2018).

[15] V.I. Orlov, E.B. Yakimov, N. Yarykin. Phys. Status Solidi C, 14 (7), 1700074 (2017)

[16] В.И. Орлов, Н.А. Ярыкин, Е.Б. Якимов. ФТП, 53 (4), 433 (2019).

[17] O.V. Feklisova, B. Pichaud, E.B. Yakimov. Phys. Status Solidi A, 202, 896 (2005).

[18] K. Wessel, H. Alexander. Phil. Mag., 35, 1523 (1977).

[19] V.G. Eremenko, E.B. Yakimov. Eur. Phys. J. Appl. Phys., 27, 349 (2004).

Редактор Л.В. Шаронова

\section{Impact of compressive and tensile deformation on a spectrum of dislocation-related luminescence in silicon}

\author{
N.A. Sobolev ${ }^{1}$, A.E. Kalyadin ${ }^{1}$, O.V. Feklisova ${ }^{2}$, \\ E.B. Yakimov ${ }^{2}$ \\ ${ }^{1}$ loffe Institute, \\ 194021 St. Petersburg, Russia \\ ${ }^{2}$ Institute of Microelectronics Technology, \\ Russian Academy of Sciences, \\ 142432 Chernogolovka, Russia
}

\begin{abstract}
Photoluminescence has been studied in silicon deformed by four-point bending at temperature of $600^{\circ} \mathrm{C}$. So-called dislocation-related luminescence lines D1, D2, D3 and D4 are observed from both the sides of the deformed samples. It is found that in the samples with the induced dislocation density $\sim 10^{7} \mathrm{~cm}^{-2}$, a luminescence intensity of the D3 and D4 lines is the same on both the sample sides, and the intensity of the D1 and D2 lines from the tensile side is higher than that from the compressive side. Behavior of the intensity of the D1 and D2 lines is well correlated with a quantity of dislocation trails. Possible

reasons of observed effect are discussed.
\end{abstract}

